\title{
The Role-Based Goal Modeling
}

\author{
Nor Ashila Abdul Rahman, Rohayanti Hassan*, Muhammad Razib Othman, Hishammuddin \\ Asmuni \\ Department of Software Engineering, Faculty of Computing, Universiti Teknologi Malaysia, 81310 UTM \\ Skudai, Johor, Malaysia, Malaysian.
}

* Corresponding author. Tel.:+0675538719; email: rohayanti@utm.my

Manuscript submitted February 23, 2016; accepted March 10, 2016.

doi: 10.17706/jsw.11.12.1242-1249

\begin{abstract}
The role-based goal model aims to innovate the existing goal model by adding the role of stakeholder element for better goal representation of system-to-be. The role-based goal model in this paper is being discussed in two aspects which are (i) on how to develop the role-based goal realization and (ii) on how to assess role-based goal realization graph. The reason for taken these two aspects into consideration is to demonstrate the differences in level of risk which later contribute to the selection of high feasibility and adequacy of requirements. There are four stages for developing the model are explained detail and include: (i) identifying of role and goal, (ii) formation of goal realization graph, (iii) assessment of confidence factor for each goal and (iv) determination of feasibility and adequacy of requirement.
\end{abstract}

Key words: Adequacy, feasibility, role-based, stakeholders.

\section{Introduction}

Concepts of goal are significant and useful in understanding and clarifying the requirement in the early stages of system developments. As in [1] describe goals as wishes, ideas and opinions from stakeholders that need to be realized in the system-to-be. Stakeholder role oriented identification is used in this process to classify the stakeholder's requirement based on the functions required in the system. Motivated from [2], goal realization graph can be used to represent the goals of system-to-be. In addition, [3] have claimed that stakeholder's representation at this level is important in order to accomplish the goals of system-to-be. With the existent of stakeholder's representation, all the stakeholder's needs that are engaged to the system could be organized wisely and produce less conflicts of unnecessary requirements from different stakeholders' point of view. Therefore, this study has innovated a new role-based goal modeling for better goal representation of system-to-be.

\section{Development of Role-Based Goal Realization Graph}

\subsection{Identifying Role and Goal}

Stakeholders may be classified under different viewpoints which represent the structuring of different stakeholders perspectives. There will be many different stakeholders with their own role and objectives [4] that need to be analyzed by requirement engineer. Reference [5] has discovered that the stakeholder identification usually focus in identifying actors for use case development rather than interactions between stakeholders. Business workflow is another useful representation that able to describe the business requirements of different stakeholders [6]. Therefore, stakeholder role of the system-to-be can be identified 
and extracted from viewpoints, use case representation and business workflow representation.

\subsection{Formation of Goal Realization Graph}

The identified goals guide the subsequent activities that influencing decisions that have to be taken during the requirement refinement. Goal graph allow hierarchical decompositions of goals. The goal realization graph uses a goal-graph as a fundamental framework that performs the KAOS (Keep All Objectives Satisfied: [7], [8]) approach, in which the requirements/objectives that need to be accomplished by the stakeholders are stated in [2]. Fig. 1 illustrates the representation of goal realization graph used in this study in a top-bottom manner. The main root of the goal represents the hard goal to be achieved by the system. Next, the goals are refined into sub goals that elaborate how the main root goal is accomplished. The sub goals are identified together with labeling its stakeholder role that able to show the importance and commitment of each stakeholder. In order to draw the goal realization graph, the following steps are required:

a) Set the most abstract hard goal as the main root goal.

b) Extend with the sub goal together with its stakeholder role: the process of subdividing into a set of goals that represent the desired system solutions and alternative to satisfy the main root goal.

c) Assign AND/OR refinement: expressing the relationship between a higher level with a lower level goal.

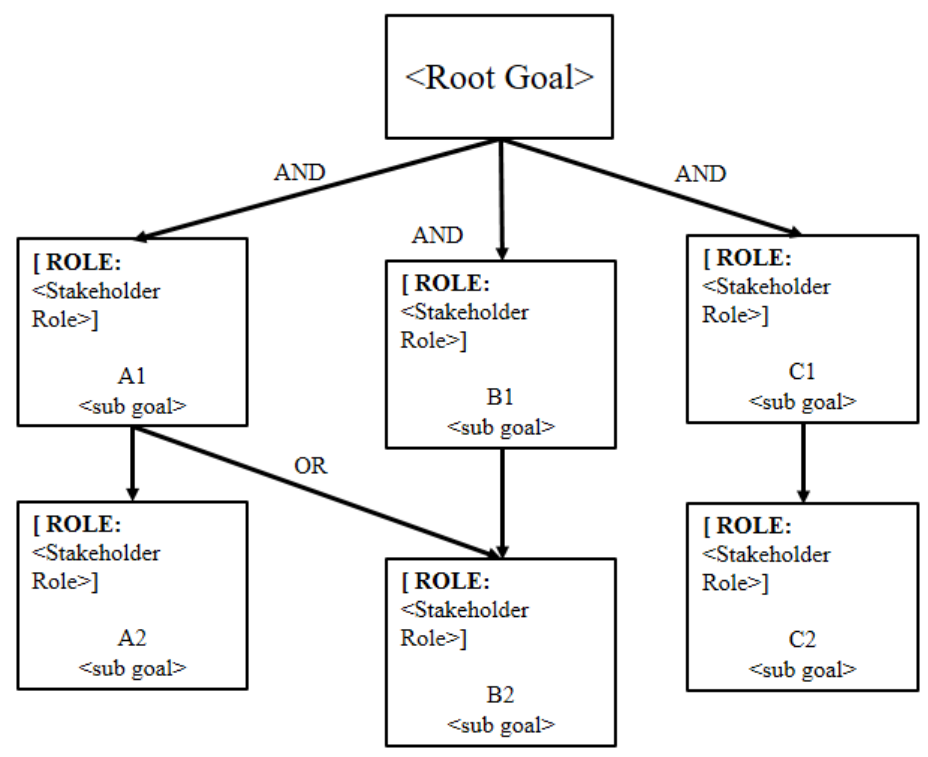

Fig. 1. A goal realization.

\subsection{Assessment of Role-Based Realization Graph}

The purpose of the goals assessment is to appraise what confidence the stakeholders might attach in the analysis expressed in the goal realization graph. In this study, four confidence factors are adopted from [2], [9] which are: (i) assumptions (ASSUME), (ii) achievability (ACHIEVE), (iii) stakeholder's mandate (MANDATE) and (iv) refinement (REFINE). The definition of chosen confidence factors are summarized in Table I. Each sub goal will be assigned with the confidence factor either ASSUME or ACHIEVE and must be assigned with MANDATE and REFINE. Once each sub goal has been assigned with the confidence factors, next each sub goal will be rated by expert using 4-point ordinal scale (NONE, LOW, MEDIUM and HIGH) as tabulated in Table II. This rating technique was adopted from [4]. The default rating for each confidence factor is Low. 
Table 1. Definition of Confidence Factor

\begin{tabular}{|c|c|}
\hline $\begin{array}{l}\text { Confidence } \\
\text { Factor }\end{array}$ & Description \\
\hline ASSUME & $\begin{array}{l}\text { The sub goal and its environment is trusted and satisfied despite the } \\
\text { stakeholder has inadequate grounds for believing this is reliable. }\end{array}$ \\
\hline ACHIEVE & $\begin{array}{l}\text { The acceptance criteria of the sub goal is achievable despite the stakeholder } \\
\text { has inadequate grounds for believing the implementation is realizable. }\end{array}$ \\
\hline MANDATE & $\begin{array}{l}\text { The sub goal is trusted and satisfied to have adequate scrutiny from the } \\
\text { stakeholder. }\end{array}$ \\
\hline REFINE & $\begin{array}{l}\text { The sub goal is still uncertain and incomplete which open to refinement from } \\
\text { the stakeholder. }\end{array}$ \\
\hline
\end{tabular}

Table 2. 4-Point Ordinal Scale Rating

\begin{tabular}{lll}
\hline \hline Rating & Description & Abbreviation \\
\hline NONE & $\begin{array}{l}\text { There is a known fact that suggest the confidence factor defined for } \\
\text { the sub goal is unsound } \\
\text { The confidence factor defined for the sub goal is questionable, or } \\
\text { the information is too fragmented or poorly corroborated to make } \\
\text { the goal more concrete }\end{array}$ & $\mathrm{L}$ \\
& $\begin{array}{l}\text { The confidence factor defined for the sub goal is believable but not } \\
\text { yet sufficient justified to warrant a higher level of rating } \\
\text { MEDIUM }\end{array}$ & $\mathrm{M}$ \\
& $\begin{array}{l}\text { The confidence factor defined for the sub goal is based on } \\
\text { high-quality information, and/or the environment of the } \\
\text { confidence factor makes it sufficiently concrete judgment }\end{array}$ & $\mathrm{H}$ \\
\hline \hline
\end{tabular}

\subsection{Determination of Feasibility and Adequacy}

According to [2], assesing the work or task is essential during requirements analysis whether it is feasible and adequate to be implemented. ASSUME and ACHIEVE confidence factors are used to estimate the feasibility of each sub goal in the goal realization graph. Meanwhile MANDATE and REFINE confidence factors are used to determine the degree of adequacy each sub goal in the goal realization graph.

As illustrated in Fig. 2, the goal realization graph was assessed from the leaves towards the root. The Feasibility (FEASIBLE) assessment is based on the weakest link technique that propagates the goal towards the root from the leaves. The FEASIBLE is then determined using both the ASSUME and ACHIEVE ratings. For instance, let say if the lowest sub goal ASSUME $=$ HIGH, ACHIEVE =MEDIUM Then the upper sub goal is determined as FEASIBLE= MEDIUM, may supplement using the following rules:

a) If lower sub goal has ASSUME $=\mathrm{LOW}$ and ACHIEVE $=\mathrm{HIGH}$ then Upper sub goal $/$ root goal has FEASIBLE $=$ LOW.

b) If lower sub goal has ASSUME = HIGH and ACHIEVE = MEDIUM then Upper sub goal/root goal has FEASIBLE $=$ MEDIUM.

c) If lower sub goal has ASSUME $=$ HIGH and ACHIEVE $=$ HIGH then Upper sub goal $/$ root goal has FEASIBLE $=$ HIGH.

As illustrated in Fig. 3, the Adequacy (ADEQUATE) assessment is calculated based on parent goal (root) towards their leaves. In [2], ADEQUATE assessment cannot be based on the combination of one single REFINE rating and one single MANDATE and should traverse from the root towards the leaves with the assumption that a possible lack of confidence in the ratings of REFINE and MANDATE. In this study, each of the sub goal has been given value of REFINE and MANDATE by expert using value of NONE, LOW, MEDIUM and HIGH. Subsequently, each sub goal is given a rating based on REFINE and MANDATE profile matrix as tabulated in Table III. For example, let say if the lowest sub goal REFINE = HIGH, MANDATE =MEDIUM Then the upper sub goal is determined as RA = MEDIUM. 


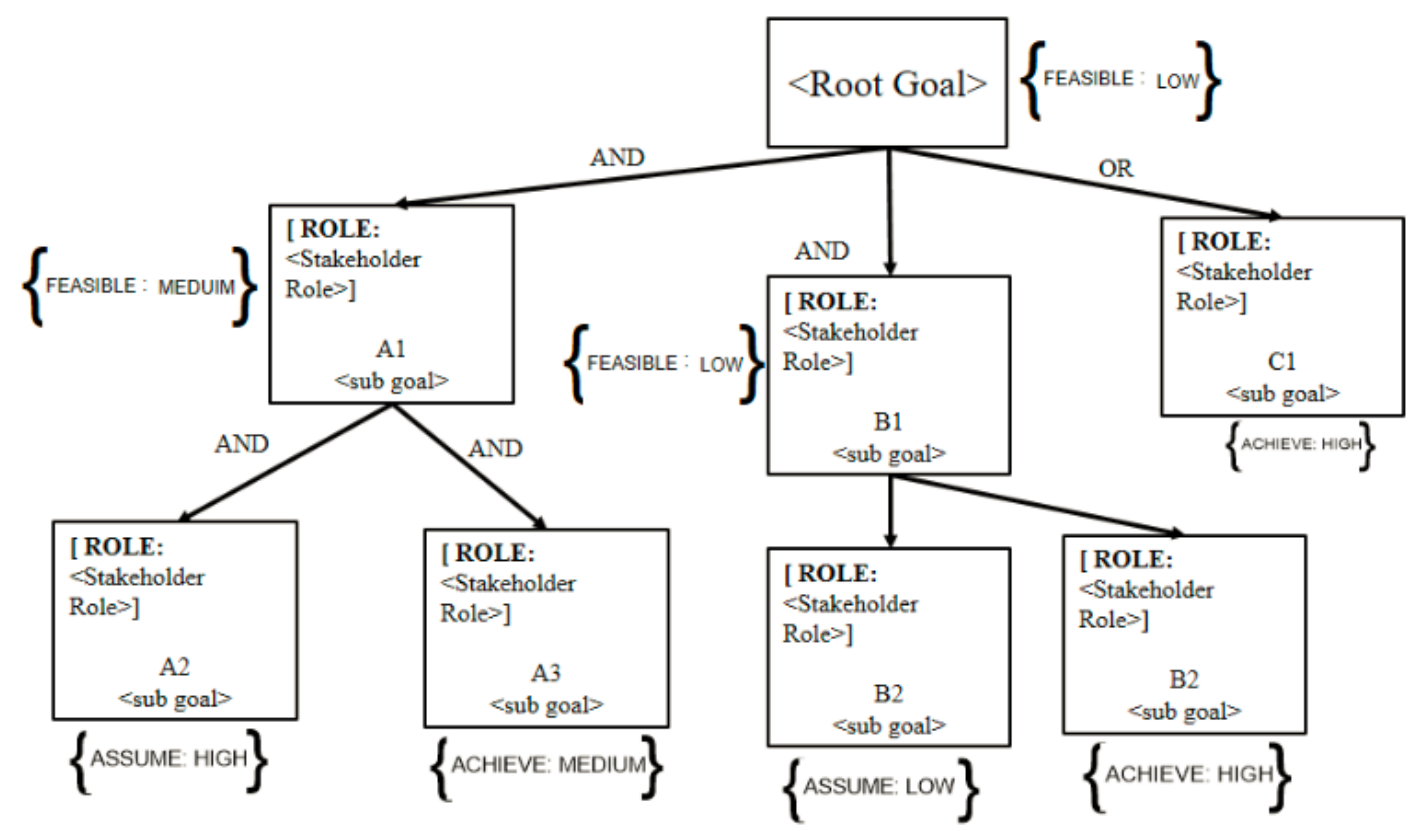

Fig. 2. The determination of FEASIBLE of goal realization graph.

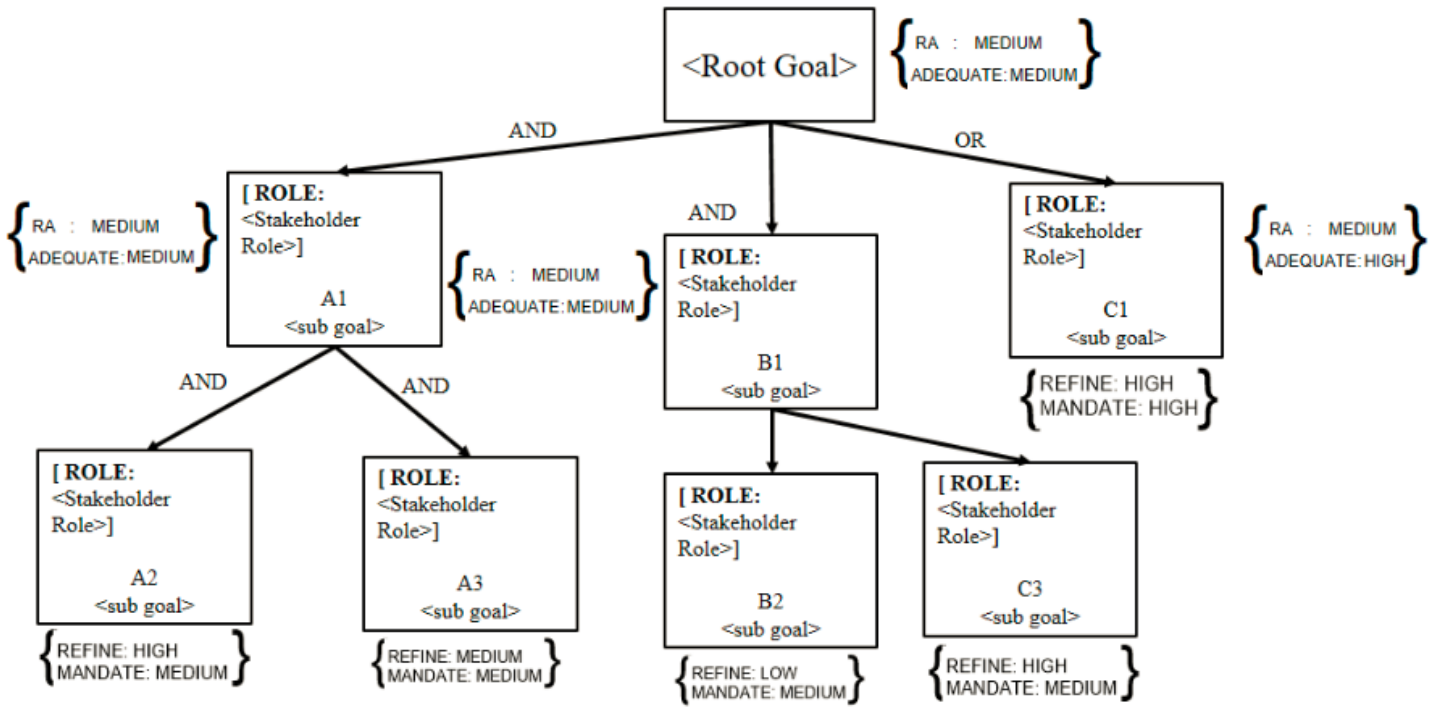

Fig. 3. The determination of ADEQUATE of goal realization graph.

Table 3. REFINE and MANDATE Profile Matrix

\begin{tabular}{|c|c|c|c|c|c|}
\hline \multirow{5}{*}{ MANDATE } & $\mathrm{H}$ & $\mathrm{N}$ & $\mathrm{L}$ & M & $\mathrm{H}$ \\
\hline & $\mathrm{M}$ & $\mathrm{N}$ & $\mathrm{L}$ & $\mathrm{L}$ & $\mathrm{M}$ \\
\hline & $\mathrm{L}$ & $\mathrm{N}$ & $\mathrm{L}$ & M & M \\
\hline & $\mathrm{N}$ & $\mathrm{N}$ & $\mathrm{N}$ & $\mathrm{N}$ & $\mathrm{N}$ \\
\hline & & $\mathrm{N}$ & $\mathrm{L}$ & $\mathrm{M}$ & $\mathrm{H}$ \\
\hline
\end{tabular}

Once the FEASIBLE and ADEQUATE ratings have been propagated through the goal realization graph, the risk of each sub goal is identified. According to [2], the higher the fraction of sub goals in the PROCEED zone, 
the lower is the risk in the requirement of the project. The CAUTION zone would be a cause for great concern due to the sub goal maybe inadequate or infeasible to be implemented. A higher number of leaf goals in the DO NOT PROCEED zone would indicate that further requirements work is required by the requirement engineer to get more information about the mandate and to re-examine the requirements. Table IV shows the risk identification as a result of value of FEASIBLE and ADEQUATE in the goal realization graph. The risk identification is determined using the following equations:

Table 4. Risk Identification

\begin{tabular}{ll}
\hline \hline Risk Rating & Description \\
\hline PROCEED & $($ ADEQUATE $=\mathrm{M} \vee \mathrm{H}) \wedge($ FEASIBLE $=\mathrm{M} \vee \mathrm{H})$ \\
CAUTION & $(($ ADEQUATE $=\mathrm{L}) \wedge($ FEASIBLE $=\mathrm{M} \vee \mathrm{H})) \vee(($ FEASIBLE $=$ \\
& L) $\wedge($ ADEQUATE $=\mathrm{M} \vee \mathrm{H}))$ \\
DO NOT & $($ ADEQUATE $=\mathrm{N} \vee \mathrm{L}) \wedge($ FEASIBLE $=\mathrm{N} \vee \mathrm{L})$ \\
PROCEED & \\
\hline \hline
\end{tabular}

\section{Implementation to the Case Study and Discussion}

The role-based goal modeling is then implemented using Plantation Integrated System (PIS) case study. PIS is an integrated, centralized and geo-distributed system that aims to manage labour management information system. This PIS system has involved multi stakeholders from multi departments and branches. Fig. 4 demonstrates a segment of business workflow in PIS system that focuses in managing labour requisition. There are five direct stakeholders (those who use the system) are identified in Fig. 4. Each role has their own goal in realizing the process of labour requisition. From this business workflow representation, the role-based goal can be modeling. All stakeholders and processes in Fig. 4 are extracted and transformed into goal realization graph as illustrated in Fig. 4 using the formation guideline described in Section 2.3 and notation guideline tabulated in Table I.

Fig. 5 presents the detail level of goal realization graph for "Manage Labour Requisition". To make the root goal more concrete, the goal is split-up into GOAL A1 and GOAL A2. GOAL A2 is refined into GOAL A2.1 and GOAL A2.1.1 in order to construct more feasibility to accomplish the root goal. Besides, GOAL A2.1.1 is made more sufficiently concrete by adding two more sub goals which are GOAL A2.1.1.1 and GOAL A2.1.1.2. For each sub goal, the role of stakeholder is attached.

Once the goal realization graph is completed design, expert rated each sub goal using the confidence factors as described in Section 2.3. In this case, experts were consists of system developer, tester and system analyst have rated the each sub goal and the assessment result is tabulated in Table V. Next, the representation of role-based goal model can be enhanced with confidence factor ratings as illustrated in Fig. 6. Based on Fig. 6, the FEASIBLE and ADEQUATE of each sub goal can be determined by observing the traversal pattern as described in Section 2.4.

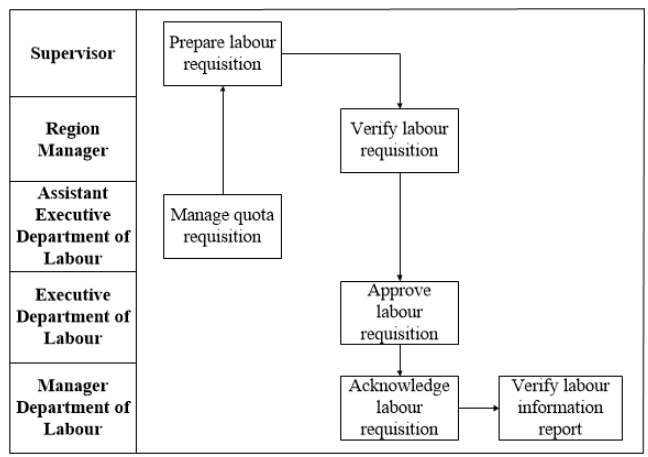

Fig. 4. A segment of business workflow in PIS system. 
Finally, the propagated FEASIBLE and ADEQUATE, the risk of each sub goal can be identified as tabulated in Table $\mathrm{V}$. Table $\mathrm{V}$ indicates that there are five goals demonstrated the PROCEED zone and two goals demonstrated CAUTION zone. It shows that in goal "Manage Labour requisition", there is minimum potential risks might attach to the requirement stated by stakeholders. If there is appearance of risk prediction, requirement engineer could re-examine the requirement before it can be proceed to the other stage of development. In addition, this role-based goal modeling gives straightforward means to derive number of requirements which are feasible or infeasible and adequate or inadequate. The risk assessment for manage labour requisition and requirements under goal GOAL A1 and GOAL 2.1.1.2 are in danger since it has been label under zone Caution. The requirement engineer or project manager has to further investigate with the stakeholder involved before it can be proceeded to the other stage of development.

Table 5. The Confidence Factor Rating

\begin{tabular}{|c|c|c|c|c|c|c|c|}
\hline \multirow{2}{*}{ Goal ID } & \multicolumn{2}{|c|}{ Confidence Factor } & & & FEASIBLE & ADEQUATE & RISK \\
\hline & ASSUME & ACHIEVE & & REFINE & MANDATE & & \\
\hline $\bar{A}$ & & HIGH & HIGH & HIGH & HIGH & HIGH & PROCEED \\
\hline A1 & MEDIUM & & MEDIUM & MEDIUM & MEDIUM & MEDIUM & CAUTION \\
\hline A2 & $\mathrm{HIGH}$ & & MEDIUM & MEDIUM & $\mathrm{HIGH}$ & HIGH & PROCEED \\
\hline A2.1 & & HIGH & MEDIUM & HIGH & HIGH & HIGH & PROCEED \\
\hline A2.1.1 & & HIGH & MEDIUM & MEDIUM & MEDIUM & HIGH & PROCEED \\
\hline A2.1.1.1 & $\mathrm{HIGH}$ & & MEDIUM & MEDIUM & MEDIUM & $\mathrm{HIGH}$ & PROCEED \\
\hline A2.1.1.2 & MEDIUM & & MEDIUM & MEDIUM & MEDIUM & MEDIUM & CAUTION \\
\hline
\end{tabular}

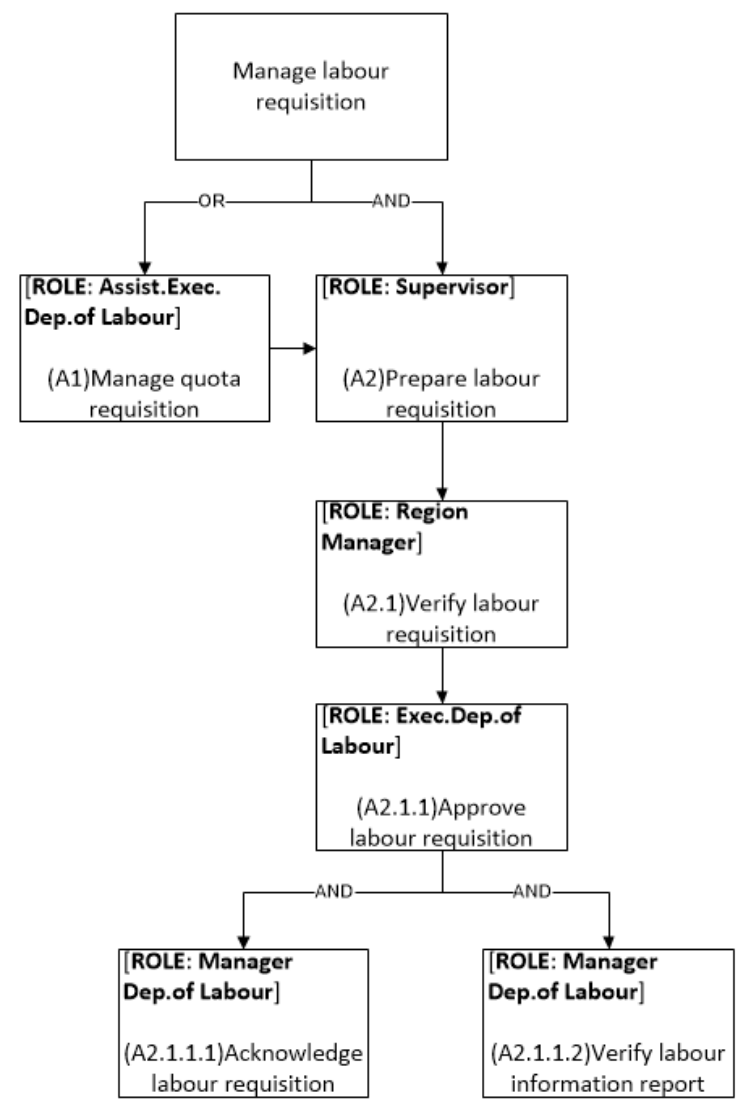

Fig. 5. The role-based goal realization graph of manage labour requisition. 


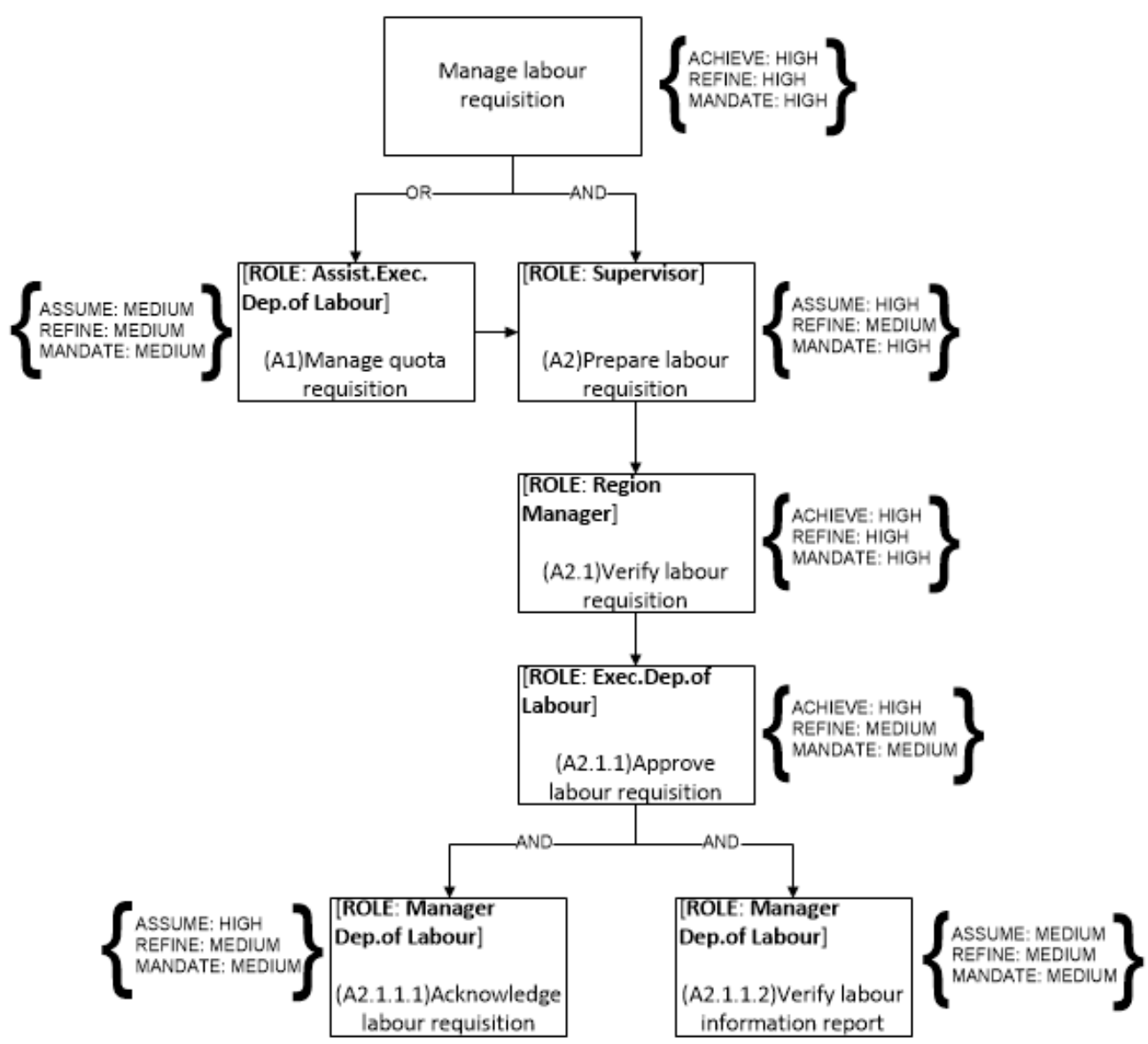

Fig. 6. The role-based goal realization graph with confidence factor rating of manage labour requisition.

\section{Conclusion}

In conclusion, this paper has described a role-based goal model which has been innovated by adding the role of stakeholder element for better goal representation of system-to-be. This paper has demonstrated on how to develop the role-based goal realization graph and followed by on how to assess the model from the aspect feasibility, adequacy and risky to facilitate the requirement engineer and project manager to analyze or estimate the severity of any requirements. For future work, the role-based goal modeling will be enhanced its representation by considering the integration element.

\section{Acknowledgment}

This research was supported by Universiti Teknologi Malaysia under Vot No. 11H84, GATES Scholars Foundation of GATES IT Solution Sdn. Bhd. and MyMaster Scholarship from Ministry of Education Malaysia. The authors highly appreciated their contributions made in term of providing project data and financial support.

\section{References}

[1] Tom, H., Monique, S., Guido, D., Antoon, G., \& Frank, S. (2013). Visualizing variability management in requirements engineering through formal concept analysis. Procedia Technology, 9(1), 189-199.

[2] Kenneth, B., Anthony, F., \& Rachel, H. (2011). A method for assessing confidence factor in requirements analysis. Information and Software Technology, 53(10), 1084-1096.

[3] Carla, P., \& Ivan, G. (2012). A systematic literature review of stakeholder identification methods in 
requirements elicitation. The Journal of Systems and Software, 85(9), 2171-2181.

[4] Zhang, Y., Harman, M., Finkelstein, A., \& Mansouri, S. A. (2011). Comparing the performance of metaheuristics for the analysis of multi-stakeholder tradeoffs in requirements optimisation. Information and Software Technology, 53(7), 761-773.

[5] Helen, S., Anthony, F., \& Galal, G., (1999). Stakeholder identification in the requirements engineering process. Proceedings of $10^{\text {th }}$ International Workshop: Database and Expert Systems Applications (pp. 387-391).

[6] Fan, S., Zhao, J. L., Dou, W., \& Liu, M. (2012). A Framework for transformation from conceptual to logical workflow models. Decision Support Systems, 54(1), 781-794.

[7] Faisal, A., \& Jeroen, K., (2010). Requirements analysis: evaluating KAOS models. Journal of Software Engineering and Applications, 3(9), 869-874.

[8] Axel van, L., (2001). Goal-Oriented requirements engineering: A guided tour. Proceedings of the 5th IEEE International Symposium on Requirements Engineering (pp. 249-262). Toronto, Canada.

[9] Kenneth, B., Anthony, F., \& Rachel, H., (2008). A lightweight technique for assessing risks in requirements analysis. IEEE Software Institution of Engineering and Technology, 2(1), 46-57.

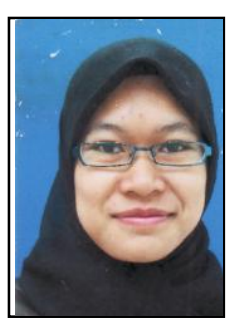

Nor Ashila Abdul Rahman is a masters student at the Department of Software Engineering in Universiti Teknologi Malaysia. She received her bachelor of computer science and she majoring in software engineering from Universiti Teknologi Malaysia in 2013. She currently continued her study in master of computer science at Universiti Teknologi Malaysia. Her research interests is related to requirements engineering and design.

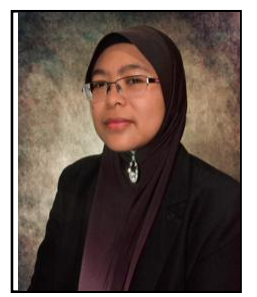

Rohayanti Hassan is a senior lecturer at the Department of Software Engineering in Universiti Teknologi Malaysia. She received her PhD in computer science from Universiti Teknologi Malaysia in 2011. Her research interests is related to requirements engineering and software testing.

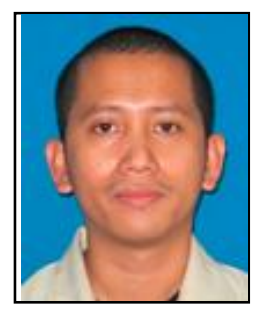

Muhamad Razib Othman is a senior lecturer at theDepartment of Software Engineering in Universiti Teknologi Malaysia. He received his $\mathrm{PhD}$ in computer science from Universiti Teknologi Malaysia in 2008. His research interest is related to artificial intelligence and machine leaning, software engineering and AGILE Unified Process (AUP).

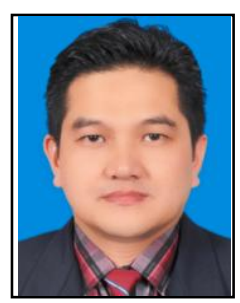

Hishammuddin Asmuni is a senior lecturer at the Department of Software Engineering in Universiti Teknologi Malaysia. He received his PhD in computer science from University of Nottingham, United Kingdom in 2008. His research interest is related to university timetabling optimization, iris biometrics and software testing. 